\title{
Optimised use of dielectric spectroscopy at microwave frequencies for direct online monitoring of polymerisation reactions.
}

\author{
Alexis Kalamiotis ${ }^{\mathrm{a}}$, Alexander Ilchev ${ }^{\mathrm{a}}$, Derek J. Irvine ${ }^{\mathrm{a}, *}$, and Georgios Dimitrakis ${ }^{\mathrm{a}, *}$ \\ ${ }^{a}$ Department of Chemical and Environmental Engineering, Faculty of Engineering, \\ University of Nottingham, Nottingham, NG7 2RD, UK
}

* Corresponding Authors at: Department of Chemical and Environmental Engineering, Faculty of Engineering, University of Nottingham, Nottingham, NG7 2RD, UK.

Tel.: +44 (0) 115 9514170. E-mail address: georgios.dimitrakis@ nottingham.ac.uk (G. Dimitrakis).

Tel.: +44 (0) 115951 4088. E-mail address: derek.irvine@ nottingham.ac.uk (D. J. Irvine)

\begin{abstract}
This study reports the first use of dielectric spectroscopy over at a wide frequency range to monitor, in real time, the progress of a ring opening polymerisation. An open-ended coaxial line sensor was placed directly into the reaction medium of a polymerisation of $\varepsilon$ caprolactone and used to characterise the dielectric properties of the polymerisation mixture both 'in-situ' and with time at microwave frequencies. In addition to measurements obtained by the sensor, samples of the medium were extracted at various time points for off-line analysis, to confirm the level of conversion and polymer molecular weights that had been achieved. The results demonstrated that the dielectric properties values exhibited by the reaction medium with time could be correlated directly to the progress of the reaction. Thus, the experimental data allowed the construction of a calibration curve which could be used to predict the conversion of monomer to polymer at any given point of the reaction. The dielectric data permitted also the identification of key reaction parameters, such as the optimum point of termination for the reaction. Furthermore, the analysis of the dielectric data over a wide frequency spectrum enabled the identification of the most suitable frequencies for the practical operation of the sensor, in terms of linearity and sensitivity. This will enable the development of suitable instrumentation and an improved strategy for the online monitoring and control of a broad range of polymerisation reactions.
\end{abstract}




\section{Keywords}

Dielectric Spectroscopy, Coaxial sensors, Online monitoring, $\varepsilon$-caprolactone, Ring opening polymerization

\section{Introduction}

Over the last 30 years, a number of novel controlled polymer synthesis methods have been developed, which allow much greater control over the final polymer structure to be achieved. These include the Nitroxide Mediated Polymerization (NMP) [1], Atom Transfer Radical Polymerization (ATRP) [2], Catalytic Chain Transfer Polymerisation (CCTP) [3], and RingOpening Polymerisation (ROP) [4]. These techniques also allow the synthesis of complex three-dimensional structures such as block, star [5], branched and hyperbranched polymers to be achieved via multi-stage process, that often require sequential addition of different monomer types [6, 7]. However, the commercial development of these complex polymers has been limited by a number of practical factors, with a key issue being the need to determine how far a particular reaction has progressed in order to continue to the next stage of the process (e.g. addition of other reactants) or terminate the reaction when the target conversion has been reached [8]. Additionally, the lack of real time feedback on reaction progress can led to batch to batch variability in large-scale products [9]. This is particularly true in the case of commercial tin catalysed ROP processes, as there known to be a variable time length induction period within the mechanism related to the formation of the catalytic species. To date, most industrial polymerisation processes involve the use of off-line techniques for molecular analysis (i.e. calculation of monomer to polymer conversion) such as Gel Permeation Chromatography (GPC) and Nuclear Magnetic Resonance (NMR) spectroscopy. These techniques require sample extraction and preparation, thus normally cannot be easily adapted to perform measurements on-line within the process and in real time. Thus, there is a growing interest to develop a direct online method for real-time monitoring the conversion of monomer to polymer, which can be easily incorporated in most industrial polymerisation reactor geometries.

Process Analytical Chemistry (PAC) techniques can be classified as off-line, at-line and online according to the type / level of sample extraction and preparation that is required [10]. Off-line and at-line analysis techniques, such as NMR and GPC, require sample extraction and transportation to a separate laboratory. By comparison, on-line analysis techniques, such as the Automated Continuous Online Monitoring of Polymerization Reactions (ACOMP), are 
performed within the process line and involve, at most, automated sampling and transport to an integrated analyser $[11,12]$. Thus, they can deliver far more relevant information that enables greater control to be exercised over the process they are monitoring. Thus, during the last few decades, many off-line techniques, such as infrared and Raman spectroscopy, have been transformed into online in order to try and control chemical processes [13]. However, when applying vibrational spectroscopic techniques, these efforts at industrial monitoring have met with limited success, with high background noise in the measurement and blackbody radiation and fluorescence in raman spectra being the most common issues [14, 15]. Recently, the use of dielectric spectroscopy for the "off-line" monitoring of chemical reactions was reported [16-18]. These studies showed that it would be possible to follow accurately the conversion of monomer to polymer by measuring the dielectric properties of the sample. However, the measurements were performed over a very limited frequency range and mainly on pre-prepared monomer to polymer concentrations. Therefore, they did not provide information on the use of the technique during the progress of a polymerisation reaction and did not consider investigating the frequencies that can potentially provide an optimum between measurement sensitivity and linearity and therefore can be utilised to inform the design and operation of dedicated sensors that can be employed as a part of a process control strategy.

In the present study, an open-ended coaxial line sensor utilised to monitor the progress of the tin octanoate mediated ring-opening polymerisation of $\varepsilon$-caprolactone (CL) over a broad frequency range $(0.5 \mathrm{GHz}-20 \mathrm{GHz})$. This work was presented on the 17 th International Meeting on Chemical Sensors, which was held in Vienna, Austria on July 2018 [19]. In this study, the dielectric properties of the reaction mixture were monitored in real time and samples were extracted at set times for off-line analysis with ${ }^{1} \mathrm{H}$ Nuclear Magnetic Resonance spectroscopy and Gel Permeation Chromatography. The dielectric response of the reaction mixture can be expressed by the complex permittivity, $\varepsilon^{*}$. The real part, also termed dielectric constant $\left(\varepsilon^{\prime}\right)$, expresses the ability of a material to store energy through polarisation, and the imaginary part, also termed dielectric loss $\left(\varepsilon^{\prime \prime}\right)$ denotes the ability of the material to dissipate the stored energy into heat. Both are frequency and temperature dependent $[20,21]$. This work investigated the relationship between the change in the dielectric properties and monomer to polymer conversion across a wide frequency range in order to identify the most suitable frequencies that can be utilised in practise to provide information regarding the progress of the polymerisation reaction. The identification of the optimum monitoring frequencies can potentially be utilised to inform the implementation of the technique and the 
design of suitable sensors and systems to accurately monitor and control such processes in the chemical industry.

\section{Materials and methods}

\subsection{Materials}

Tin(II) 2-ethylhexanoate (96\%) (Sn(Oct)2), $\varepsilon$-caprolactone (99\%), and benzyl alcohol (99\%) $(\mathrm{BzOH})$ were all purchased from Alfa Aesar and used as received without any purification.

\subsection{Ring Opening Polymerisation Procedure}

All experiments were conducted with a constant $[\mathrm{CL}]:\left[\mathrm{Sn}(\mathrm{Oct})_{2}\right]:[\mathrm{BzOH}]$ molar ratio of $800: 1: 10 . \mathrm{Sn}(\mathrm{Oct})_{2}$ was chosen as the preferable catalyst because it is used in the industrial production of polycaprolactone [22]. The most favoured mechanism for the ROP of CL using $\mathrm{Sn}(\mathrm{Oct})_{2}$ that is reported in the literature is the coordination-insertion mechanism (Fig.1), in which the catalyst adds to the hydroxyl groups of the initiator and forms an alkoxide bond [23].
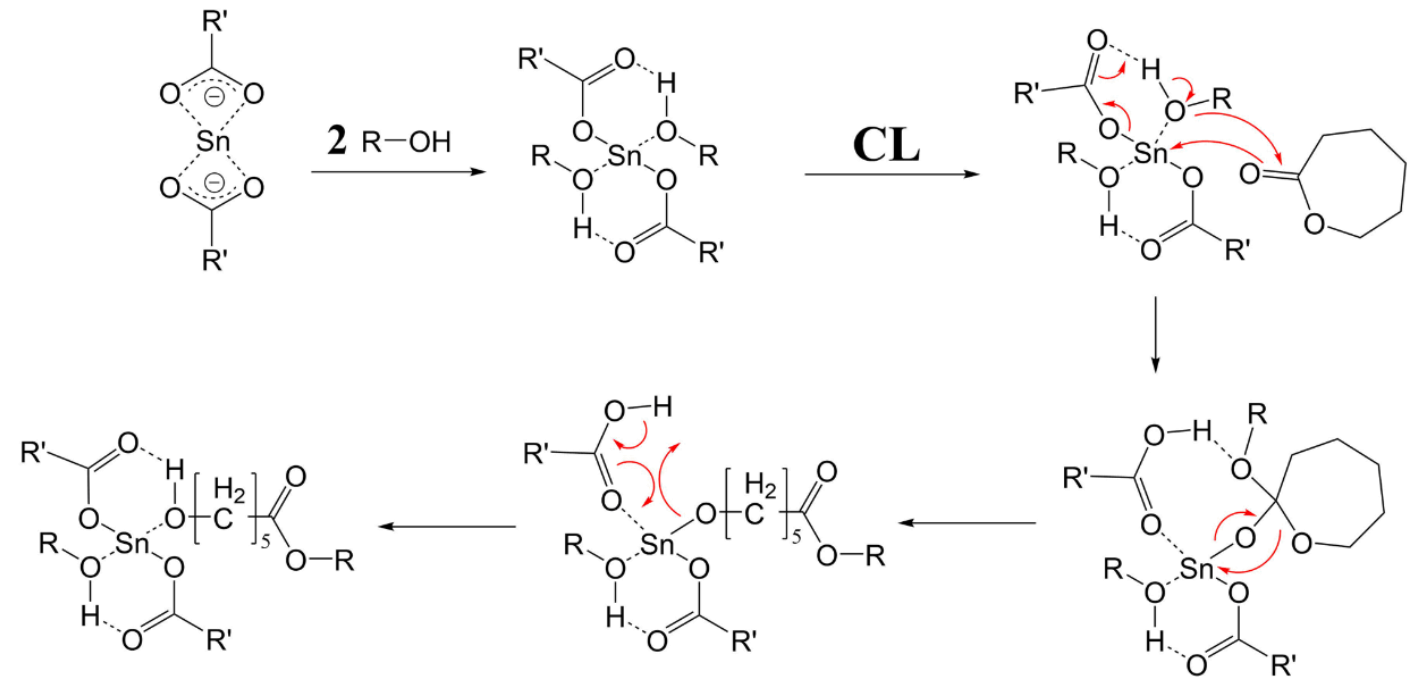

Fig. 1. $\mathrm{Sn}(\mathrm{Oct})_{2}$ catalysed coordination-insertion ROP mechanism of CL [24].

The reaction mixture tin(II) 2-ethylhexanoate $(0.13 \mathrm{~g}, 0.32 \mathrm{mmol}), \varepsilon$-caprolactone $(29.52 \mathrm{~g}$, $0.26 \mathrm{~mol})$, and benzyl alcohol $(0.35 \mathrm{~g}, 3.24 \mathrm{mmol})$ was placed into a $50 \mathrm{~mL}$ flask and prepurged with nitrogen for 15 minutes. The mixture was then heated to the target reaction temperature $\left(160^{\circ} \mathrm{C}\right)$ with the use of a preheated oil bath and the mixture was mechanically stirred throughout the reaction progress. The reaction mixture temperature was monitored using a thermocouple. An open-ended coaxial line sensor was used to monitor the dielectric 
properties during the polymerisation progress. At set times during the polymerisation progress, samples were extracted using a Hamilton syringe and needle and placed in a refrigerator, for further off-line analysis with Nuclear Magnetic Resonance spectroscopy. At the end of the reaction, the flask was removed, and crude product was also analysed with NMR and GPC.

\subsection{On-line Dielectric Characterization Method}

Open ended coaxial sensor design: The dielectric property measurement during the polymerisation progress was conducted with the use of an Agilent $85070 \mathrm{E}$ open ended coaxial line sensor and a Keysight N5232A PNA-L Vector Network Analyser (VNA) over the frequency range of $0.5 \mathrm{GHz}$ to $20 \mathrm{GHz}$ changing in $50 \mathrm{MHz}$ increments, as shown in Figure 2. The coaxial sensor was made of stainless steel (T316L body and T304L tip) with a dielectric insert was made of borosilicate glass. The construction of the sensor took the form of a $9.5 \mathrm{~mm}$ coaxial line with an inner nickel-plated tungsten center conductor of $1.6 \mathrm{~mm}$ and was capable of operating in the temperature range from $-40{ }^{\circ} \mathrm{C}$ up to $200{ }^{\circ} \mathrm{C}$ [25].
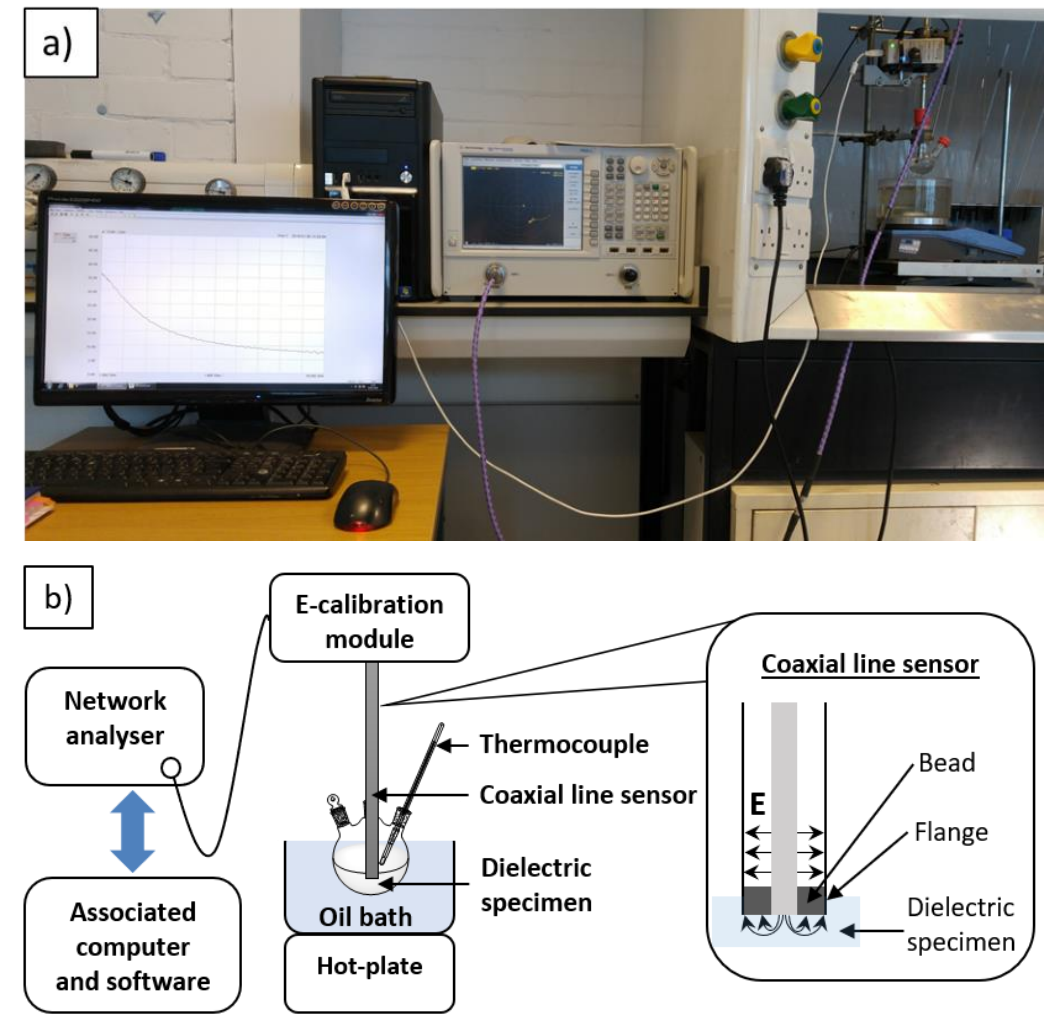

Fig. 2. a) Experimental setup and apparatus, b) Schematic diagram of experimental set up and coaxial line sensor where also the E-field orientation of the TEM propagating wave (in the sensor) is shown. 
“On-line” Polymerisation Measurements: The open-ended coaxial line sensor was introduced to the flack through a rubber septum, such that the end was immersed into the reaction mixture. A swept signal $(0.5 \mathrm{GHz}-20 \mathrm{GHz})$ was then transmitted from the VNA, through the open-ended coaxial line, into the sample. Depending on the complex permittivity of the sample, a portion of that signal was reflected to the VNA and the reflection $\left(\mathrm{S}_{11}\right)$ coefficient of the sample were collected and stored on a dedicated PC. The complex permittivity of the sample was determined using Nicolson-Ross-Weir method using reflection and transmission coefficients [26, 27].

Calibration of Apparatus: This method of dielectric property assessment requires calibration prior to the beginning of a polymerisation experiment, in order to account for systematic errors/experimental variation. For the purpose of calibration, a least-squares calibration algorithm was selected, and three standards were used: open line (air), short-circuited line (calibration short tool) and a reference liquid of known dielectric response (water). This method is the most commonly used calibration procedure detailed in the literature [28]. The quality of calibration was assessed by evaluating the residual calibration errors over the entire frequency range used (500 MHz to $20 \mathrm{GHz}$ ), which includes the frequencies at which we have presented the dielectric property data [29]. The calibration was repeated if the residual errors were larger than $2 \%$. As an additional step to ensure the quality of calibration, water and methanol samples were measured and their dielectric properties were compared with the literature [30].

\subsection{Off-line Molecular Analysis}

The extracted samples were subjected to off-line analysis by Proton Nuclear Magnetic Resonance spectroscopy $\left({ }^{1} \mathrm{H}\right.$ NMR) and Gel Permeation Chromatography (GPC). This analysis enabled the number average molecular weight $\left(\mathrm{M}_{\mathrm{n}}\right)$, dispersity $(\mathrm{Ð})$, and the mole ratio between monomer and polymer (i.e. level of conversion) to be calculated.

Nuclear Magnetic Resonance spectroscopy (NMR): ${ }^{1} \mathrm{H}$ NMR spectra were recorded at $25{ }^{\circ} \mathrm{C}$ on a Bruker AVIIIHD 400 (400 MHz) spectrometer, equipped with cryoprobe to prevent further reaction during analysis. The samples were dissolved in deuterated chloroform $\left(\mathrm{CDCl}_{3}\right)$ to which the chemical shifts were referenced $(7.26 \mathrm{ppm})$. The analysis of the results was performed using the Mnova ${ }^{\circledR}$ software package from Mestrelab Research. Conversion 
was determined by comparing the integral of the proton resonance of the methylene moiety adjacent to oxygen of the carbonyl group for both the monomer and polymer. The number average molecular weight was determined by comparing the integral of methylene proton resonance adjacent to the carbonyl group to that of methylene proton belonging to the benzyl ester end group.

Gel Permeation Chromatography (GPC): GPC analysis was performed on an Agilent 1260 infinity series HPLC with an online vacuum degasser, an Agilent online differential refractometer (DRI) and a Wyatt Heleos II Multi-Angle Light Scattering detector (MALS). Two Agilent $5 \mu \mathrm{m}$ PLgel Mixed C columns $(7.5 \times 300 \mathrm{~mm})$ were connected in series with an Agilent PLgel $5 \mu \mathrm{m}$ Guard column $(7.5$ x $50 \mathrm{~mm})$. The column oven, DRI and MALS temperature were set to $35^{\circ} \mathrm{C}$ and the flow rate was set to $1 \mathrm{~mL}$ per minute. Each sample was dissolved in HPLC grade tetrahydrofuran (THF), at a concentration of $1 \mathrm{mg} / \mathrm{mL}$. DRI calibration was performed using $30 \mathrm{kDa}$ and $200 \mathrm{kDa}$ linear polystyrene narrow dispersity standards dissolved in THF at a concentration of $1.0 \mathrm{mg} / \mathrm{mL}$. MALS calibration was performed using HPLC grade toluene, filtered through a $0.02 \mu \mathrm{m}$ PTFE filter and normalisation was performed in THF using a $30 \mathrm{kDa}$ linear polystyrene narrow dispersity GPC calibration standard. The columns were calibrated using narrow dispersity polystyrene standards ranging from $580 \mathrm{Da}$ to $1000 \mathrm{kDa}$.

\section{Results and Discussion}

\subsection{Dielectric properties of individual precursors and reaction mixtures}

The dielectric properties of the monomer $(\mathrm{CL})$, catalyst $\left(\mathrm{Sn}(\mathrm{Oct})_{2}\right)$, initiator $(\mathrm{BzOH})$, and polymer (PCL) were measured using the described open ended coaxial line sensor to highlight the differences between the dielectric response of the individual precursors and the final product. A broad frequency range $(500 \mathrm{MHz}-20 \mathrm{GHz})$ was investigated in $50 \mathrm{MHz}$ increment steps. The dielectric properties of the monomer were found to be higher than that of its related polymer, as it has been previously reported [17, 18]. The dielectric properties of catalyst and initiator were assessed and found to have only minor contribution to the polymerisation mixture at the target reaction temperature $\left(160^{\circ} \mathrm{C}\right)$ and concentration that they are present in the reaction mixture, i.e. the selected monomer:catalyst:initiator molar ratio (800:1:10), which is consistent with previous studies [17, 18]. Furthermore, the tin mediated ROP polymerisation is a chain growth polymerisation which exhibits pseudo living features (i.e. linear increase of molecular weight with conversion) [31]. As the chain forms, 
the number of degrees of molecular freedom within each repeat unit is reduced compare to those of the monomer. Thus the dielectric properties of the mixture have been found to decrease as the reaction progresses and this could be possibly attributed to the consumption of the high dielectric properties monomer during its conversion to the low dielectric properties polymer as has been previously suggested [18].

The data in Figure 3 and 4 shows how the dielectric constant and loss of $\varepsilon$-caprolactone monomer and polymer mixtures vary with frequency when measured by the coaxial probe method. Both dielectric constant and loss drop significantly with increasing amount of polymer, which is in agreement with previously published cavity perturbation data [16-18].

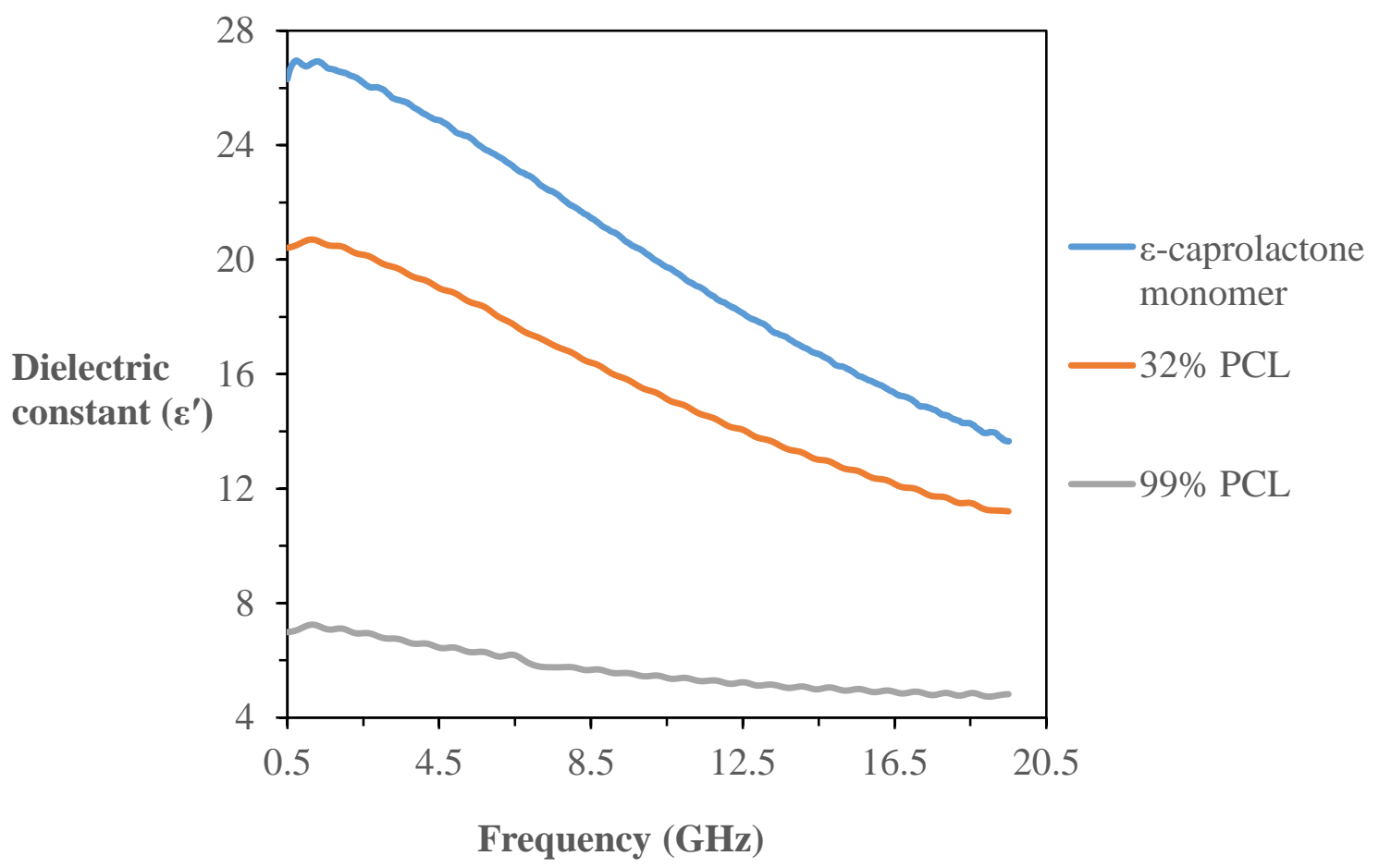

Fig. 3. Plot of the dielectric constant versus frequency $(\mathrm{GHz})$ of $\varepsilon$-caprolactone monomer and polymer mixtures at $160{ }^{\circ} \mathrm{C}$. The maximum standard deviations of the measured data are: $\varepsilon$ caprolactone monomer: [ $\left.\varepsilon^{\prime} \pm 0.3\right]$; 32\% PCL: [ $\left.\varepsilon^{\prime} \pm 0.4\right]$; 99\% PCL: $\left[\varepsilon^{\prime} \pm 0.3\right]$. 


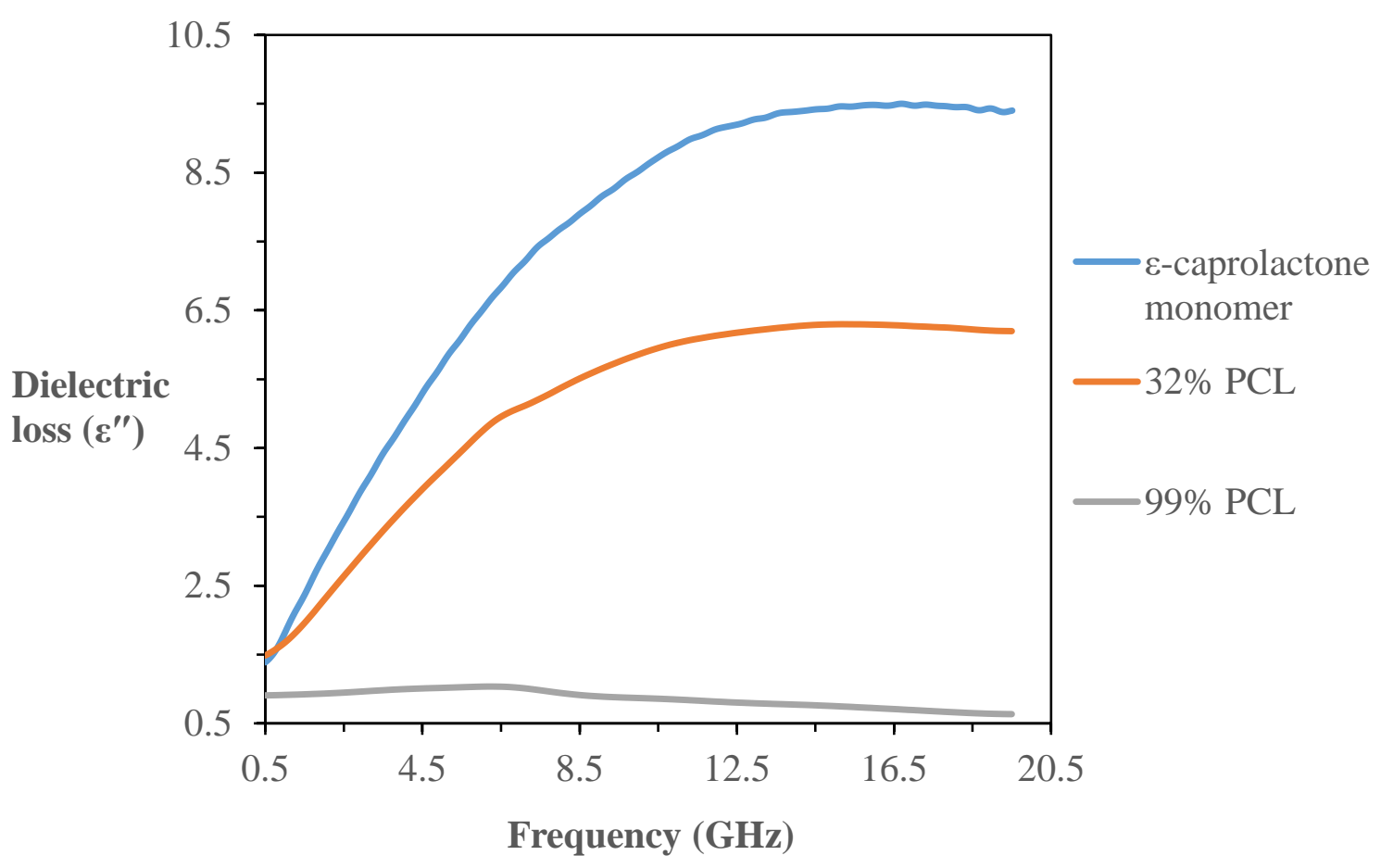

Fig. 4. Plot of dielectric loss versus frequency $(\mathrm{GHz})$ of $\varepsilon$-caprolactone monomer and polymer mixtures at $160^{\circ} \mathrm{C}$. The maximum standard deviations of the measured data are: $\varepsilon$-caprolactone monomer: [ $\left.\varepsilon^{\prime \prime} \pm 0.2\right]$; $32 \%$ PCL: [ $\left.\varepsilon^{\prime \prime} \pm 0.2\right]$; 99\% PCL: [ $\left.\varepsilon^{\prime \prime} \pm 0.1\right]$.

\subsection{Frequency dependence of the Dielectric properties during the polymerisation reaction}

The dielectric properties of the reaction mixture were measured over a broad frequency range at set times during the progress of the polymerisation. At each time point, a swept frequency signal from $500 \mathrm{MHz}$ up to $20 \mathrm{GHz}$ in $50 \mathrm{MHz}$ steps was transmitted from the Vector Network Analyser into the sample, via the coaxial line, and the dielectric properties were calculated based on the reflection coefficient of the sample, as described in section 2.4. Figure 5 and 6 show how the dielectric constant and loss varied with frequency at selected time points during the reaction. Compared to the prior published results [16-18], this data was collected "on-line" within the reaction mixture during the polymerisation process in real time and over a much broader frequency range. 


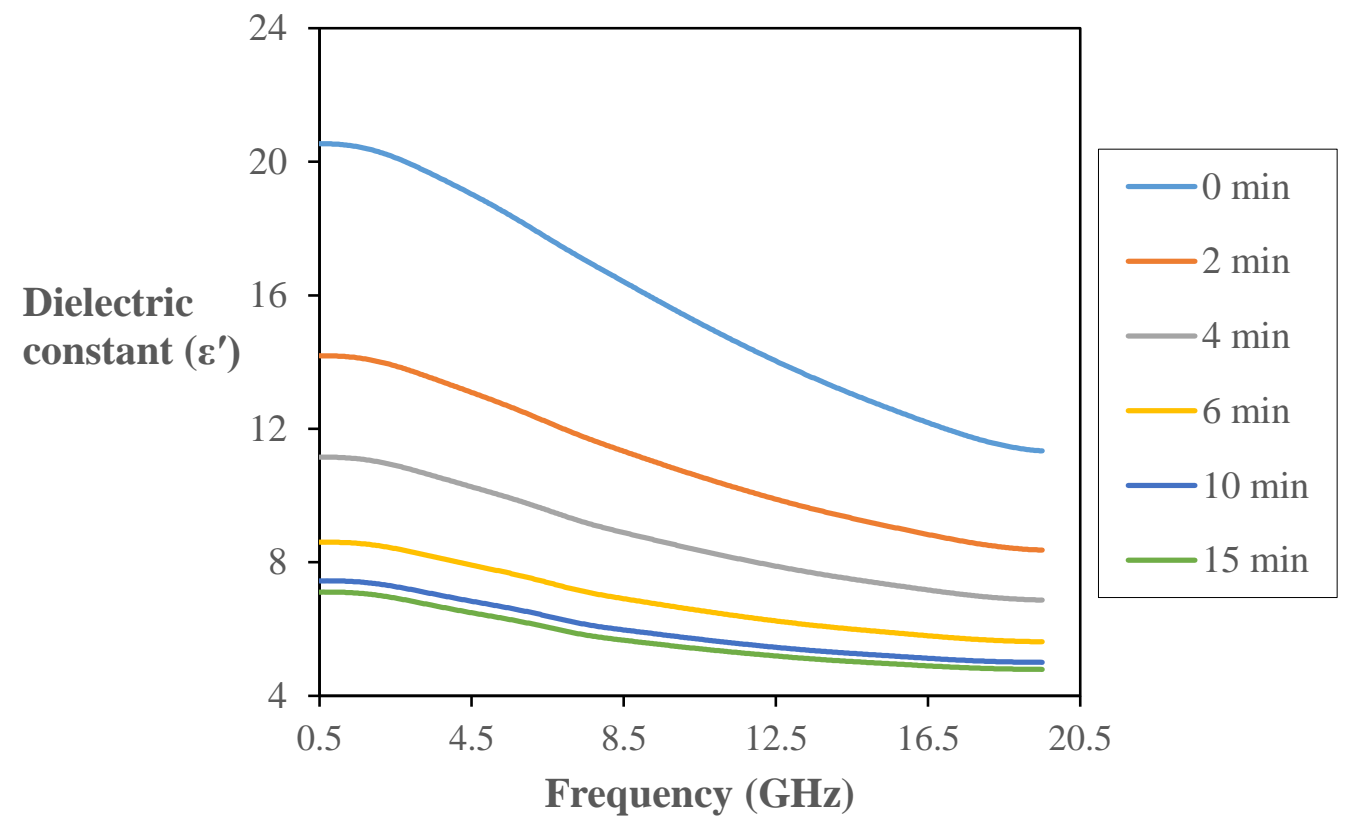

Fig. 5. Plot of dielectric constant versus frequency $(\mathrm{GHz})$ at fixed time points during the polymerisation of $\varepsilon$-caprolactone at $160{ }^{\circ} \mathrm{C}$. The maximum standard deviations of the measured data are: 0 min: $\left[\varepsilon^{\prime} \pm 0.5\right] ; 2$ min: $\left[\varepsilon^{\prime} \pm 0.6\right] ; 4 \min :\left[\varepsilon^{\prime} \pm 0.5\right] ; 6 \mathrm{~min}:\left[\varepsilon^{\prime} \pm 0.4\right] ; 10$ min: $\left[\varepsilon^{\prime} \pm 0.3\right]$; 15 min: $\left[\varepsilon^{\prime} \pm 0.2\right]$.

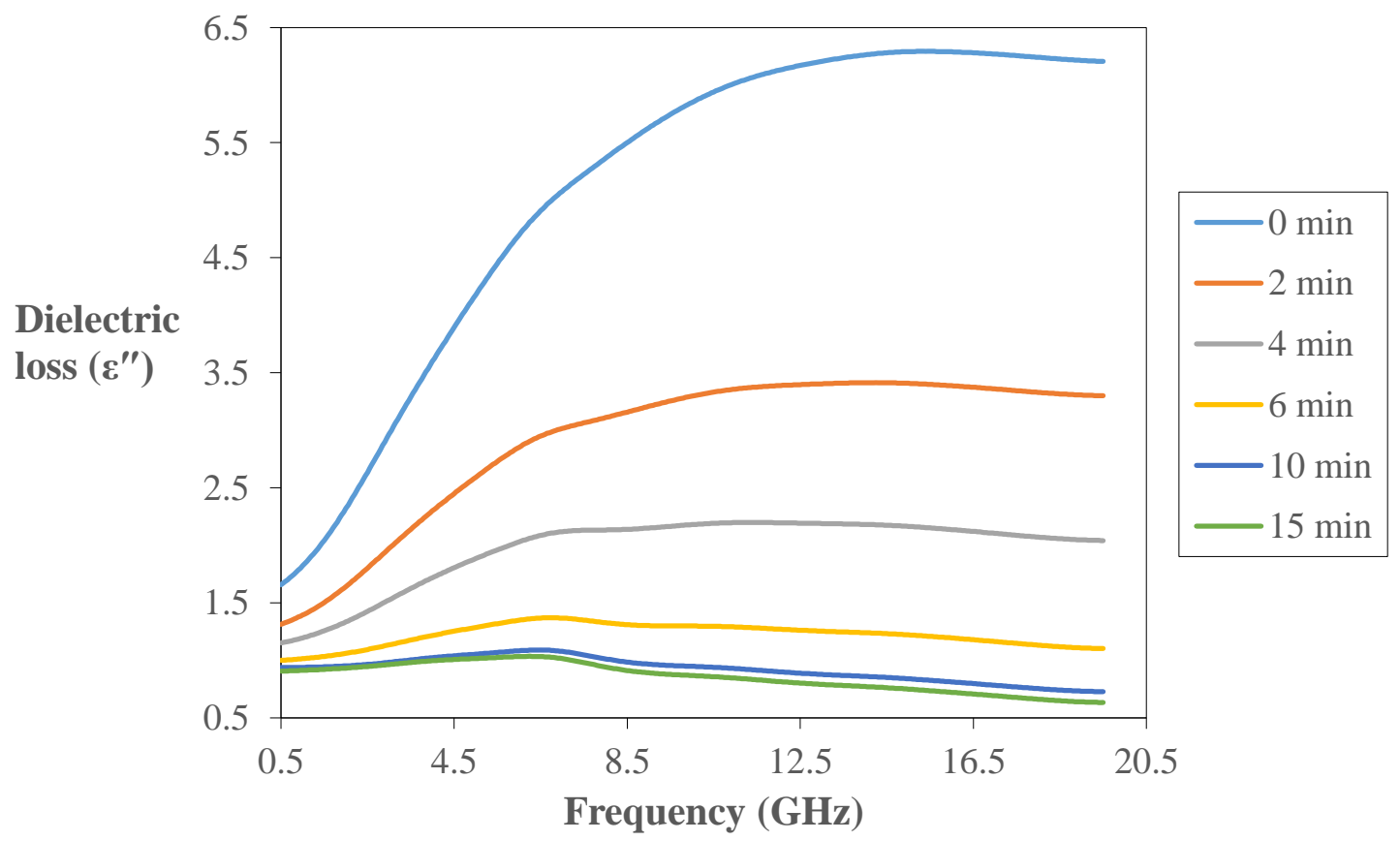

Fig. 6. Plot of dielectric loss versus frequency $(\mathrm{GHz})$ at fixed time points during the polymerisation of $\varepsilon$-caprolactone at $160{ }^{\circ} \mathrm{C}$. The maximum standard deviations of the measured data are: 0 min: [ $\left.\varepsilon^{\prime \prime} \pm 0.2\right] ; 2$ min: $\left[\varepsilon^{\prime \prime} \pm 0.2\right] ; 4$ min: $\left[\varepsilon^{\prime \prime} \pm 0.2\right] ; 6$ min: $\left[\varepsilon^{\prime \prime} \pm 0.2\right] ; 10$ min: $\left[\varepsilon^{\prime \prime} \pm 0.1\right]$; 15 min: $\left[\varepsilon^{\prime \prime} \pm 0.1\right]$.

In Figures 5 and 6 , both $\varepsilon^{\prime}$ and $\varepsilon^{\prime \prime}$ were observed to decrease during the progress of the polymerisation across the whole frequency range investigated. Meanwhile, the comparison of 
the dielectric data at low and high frequencies enabled the identification of the most suitable frequency for the monitoring of this particular chemical transformation. In Figure 5, $\varepsilon^{\prime}$ exhibits the largest decrease during the polymerisation progress at low frequencies. For example, $\varepsilon^{\prime}$ at $0.5 \mathrm{GHz}$ decreases from 20.5 to 11.3 as the polymerisation time increases from 0 min to $15 \mathrm{~min}$, whilst at $19 \mathrm{GHz}$, a smaller decrease of 11.4 to 4.8 was recorded. However, the opposite trend was observed in $\varepsilon^{\prime \prime}$. In Figure $6, \varepsilon^{\prime \prime}$ values at $19 \mathrm{GHz}$ decreased from 6.2 to 0.6 while at $0.5 \mathrm{GHz}$ decreased from 1.7 to 0.9 . As the polymerisation reaction is progressing, the relaxation frequency of the system exhibits a broadening and a shift towards lower frequencies, this likely relates to the presence of the polymer which possesses a higher relaxation time and also the presence of intermolecular/inter-chain interactions [17]. For polymerisation times larger than 6 minutes a small resonant peak is observed at approximately $6.5 \mathrm{GHz}$. The origin of that behaviour is unclear at the moment and requires further investigation. However, the possibility of this observation being simply an experimental artefact cannot be excluded as the open ended coaxial probe technique has a number of known limitations [28-30] in characterising low dielectric loss materials. The largest scale measurement changes for $\varepsilon^{\prime}$ and $\varepsilon^{\prime \prime}$ were found at frequencies $0.95 \mathrm{GHz}$ and $18.35 \mathrm{GHz}$ respectively. The use of both, real and imaginary parts of the permittivity can enable the selection of the most appropriate parameter that exhibits better linearity, depending on the conditions of the reaction, in order to construct the most accurate calibration curve for monitoring the monomer to polymer conversion. Depending on the system under study and the process conditions a careful selection of the most appropriate type of sensor is required. For systems exhibiting large values of $\varepsilon^{\prime}$ and $\varepsilon^{\prime \prime}$ open ended coaxial line techniques are expected to perform well provided that a good contact between the sensor and the material is maintained (i.e. presence of bubbles, turbulence is avoided). In cases where the system under study exhibits low values of $\varepsilon^{\prime}$ and $\varepsilon^{\prime \prime}$ the use of resonant techniques is expected to produce more accurate results provided that adequate provisions are in place to introduce the material in the sensor.

\subsection{Relationship between the Dielectric Properties and Monomer to Polymer Conversion}

It has been reported that this reaction mechanism exhibits pseudo living characteristics i.e. a linear conversion of monomer to polymer with time $[17,18]$. Figure 7 shows a comparison of $\varepsilon^{\prime}$ and number average molecular weight $\left(\mathrm{M}_{\mathrm{n}}\right)$ versus monomer to polymer conversion. Each data point represents the average of 5 measurements and the standard deviation was used to estimate the error. 


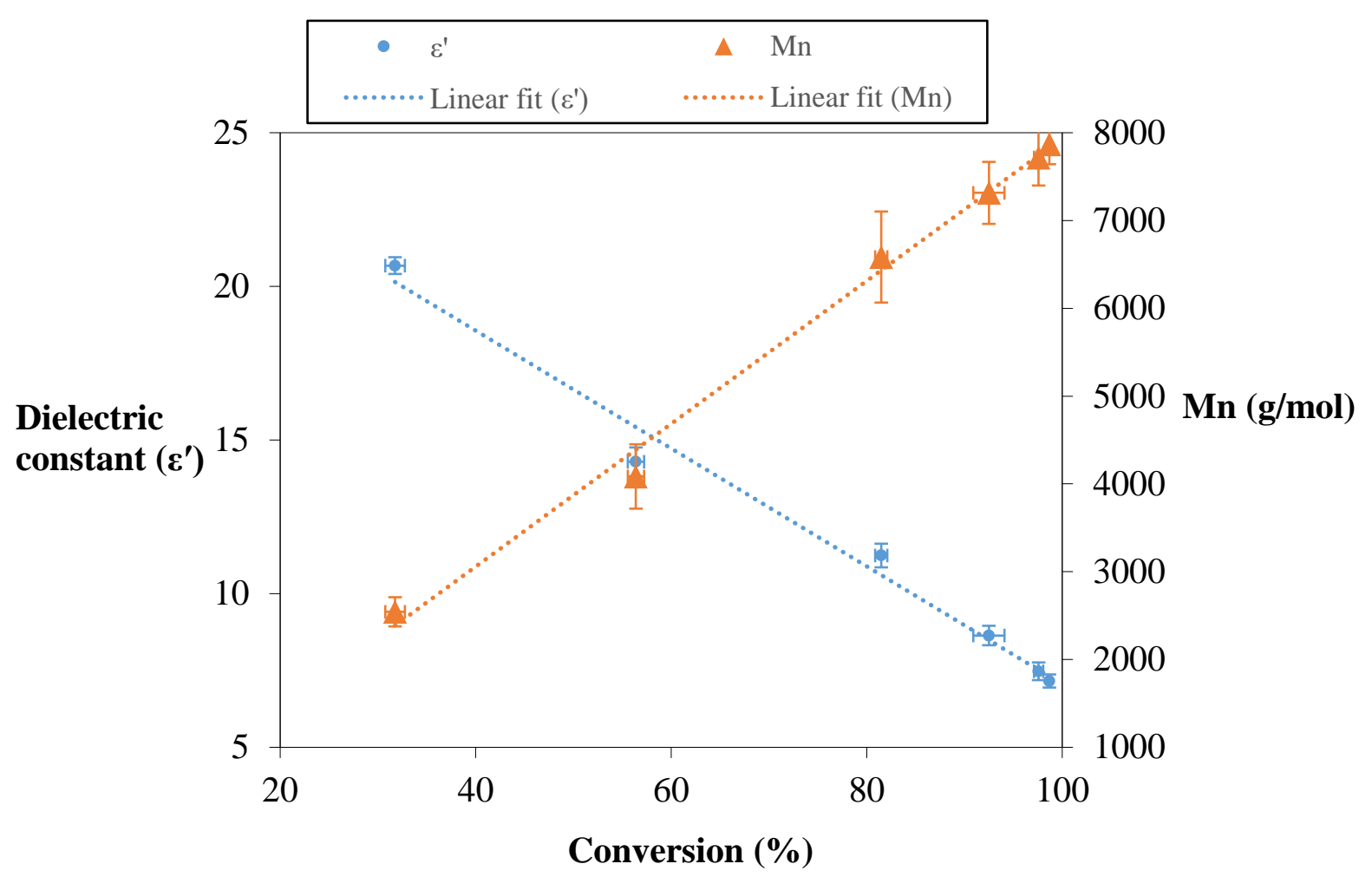

Fig. 7. Comparison plot of dielectric constant $\left(\varepsilon^{\prime}\right)$ at $0.95 \mathrm{GHz}$ and number average molecular weight $(\mathrm{g} / \mathrm{mol})$ versus monomer to polymer conversion $(\%)$.

In this case, the dielectric data has been generated by "on-line" probe measurements, whilst the conversion data was determined with "off-line" NMR spectroscopy. As the $M_{n}$ increases during the progress of the polymerisation, the dielectric properties exhibit a mirrored linear decrease. In detail, $\varepsilon^{\prime}$ decreases from 20.7 to 7.1 after 15 minutes of polymerisation time while the $\mathrm{M}_{\mathrm{n}}$ increases from 2543 to $7869 \mathrm{~g} \mathrm{~mol}^{-1}$. This relates to a monomer to polymer conversion from $32 \%$ to $99 \%$ which was also determined by NMR. As the monomer consumption is directly proportional to polymer growth a theoretical molecular weight can be predicted from the initial relative concentration of the monomer and the initiator [32]. The conversion corrected $\mathrm{M}_{\mathrm{n}}$ values for this polymer were found to be in close agreement with the calculated, theoretical $M_{n}$ values (see Table 1). Thus, this demonstrated that the polymerisation was exhibiting high levels of control with the probe inserted in the reaction mixture. Therefore, the data in Fig. 6 demonstrates that; (a) the dielectric probe data can be used to record the loss of monomer during the polymerisation [18], and (b) a well-controlled polymerisation can be conducted with the probe in place. As a result, it was concluded that an open-ended coaxial line sensor can be used to monitor the progress of the $\varepsilon$-caprolactone ROP polymerisation. 
Table 1: Experimental conditions, theoretical and observed $\mathrm{Mn}$ and Dispersity values for the bulk polymerisation of $\varepsilon$-caprolactone using benzyl alcohol and tin octanoate at $160{ }^{\circ} \mathrm{C}$.

\begin{tabular}{|c|c|c|c|c|}
\hline $\begin{array}{c}\text { Conversion }^{\mathbf{a}} \\
(\%)\end{array}$ & $\begin{array}{c}\text { Mn,Theory } \\
\text { (g/mol) }\end{array}$ & $\begin{array}{c}\mathrm{Mn}, \mathrm{NMR}^{\mathrm{a}} \\
(\mathrm{g} / \mathrm{mol})\end{array}$ & $\begin{array}{c}\mathrm{Mn}_{\mathrm{n}, G P C}{ }^{\mathrm{b}} \\
(\mathrm{g} / \mathrm{mol})\end{array}$ & $\boldsymbol{\boxplus}^{\mathbf{b}}$ \\
\hline 32 & 3000 & 2500 & 2700 & 1.16 \\
\hline 56 & 5200 & 4100 & 4000 & 1.29 \\
\hline 82 & 7500 & 6600 & 6300 & 1.48 \\
\hline 93 & 8500 & 7300 & 6800 & 1.71 \\
\hline 98 & 9100 & 7700 & 7100 & 1.91 \\
\hline 99 & 9100 & 7900 & 6800 & 2.14 \\
\hline
\end{tabular}

${ }^{\mathrm{a}}$ Determined using ${ }^{1} \mathrm{H}$ NMR $\left(400 \mathrm{MHz}, \mathrm{CDCL}_{3}, 25^{\circ} \mathrm{C}\right)$.

${ }^{\mathrm{b}}$ Determined using GPC calibrated with polystyrene standards.

There was good agreement between the NMR and GPC generated molecular weight data showing that there was a clear evidence of growth with time that was in line with the predicted growth that was predicted by the theoretically calculated values. This was evidence that a well-controlled polymerisation had been achieved. The differences between the theoretical and experimental values were as a result of water present in the monomer acting as an initiator. Drying of the monomer should result in reduction/removal of this offset.

\subsection{Construction of a calibration curve for monitoring online the $\varepsilon$-caprolactone polymerisation}

As it has been previously discussed, both $\varepsilon^{\prime}$ and $\varepsilon^{\prime \prime}$ decreased during the polymerisation across the frequency range investigated $(0.5 \mathrm{GHz}-20 \mathrm{GHz})$ and the largest scale measurement changes, which also exhibited the best linearity, for $\varepsilon^{\prime}$ and $\varepsilon^{\prime \prime}$ were found at frequencies $0.95 \mathrm{GHz}$ and $18.35 \mathrm{GHz}$ respectively. Therefore, in order to utilise dielectric properties to follow the conversion of monomer to polymer in the $\varepsilon$-caprolactone polymerisation in a systematic way, a calibration curve was constructed using a method detailed in the literature [17]. Figures 8 and 9 show the trend on the $\varepsilon^{\prime}$ and $\varepsilon^{\prime \prime}$ at frequency $0.95 \mathrm{GHz}$ and $18.35 \mathrm{GHz}$ as the conversion ranges from $32 \%$ to $99 \%$. Each data point in the following figures is the average of five measurements. The error bars in each data point represent the standard deviation. 


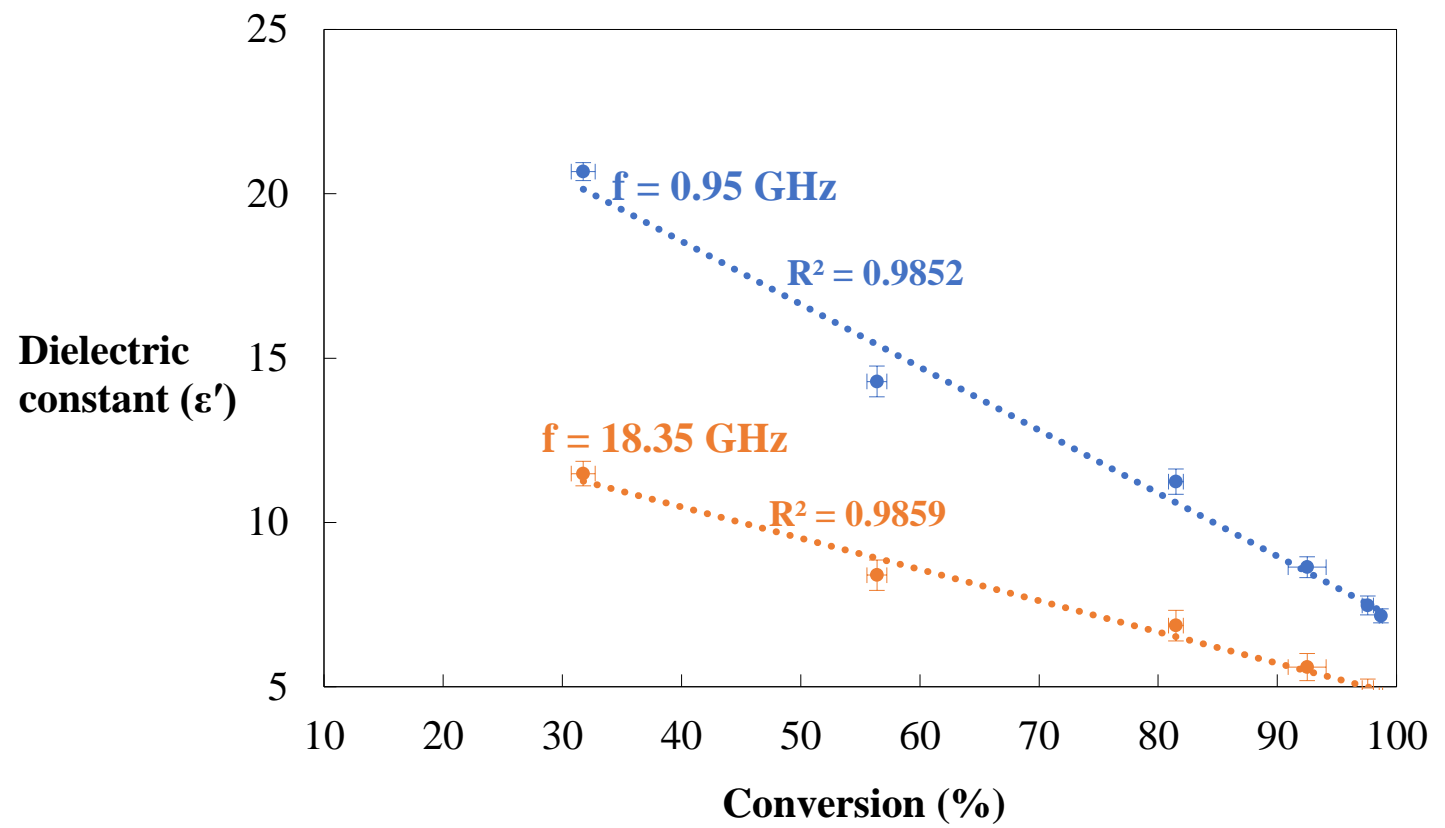

Fig. 8. Graph of dielectric constant $\left(\varepsilon^{\prime}\right)$ versus conversion at the frequencies $0.95 \mathrm{GHz}$ and $18.35 \mathrm{GHz}$.

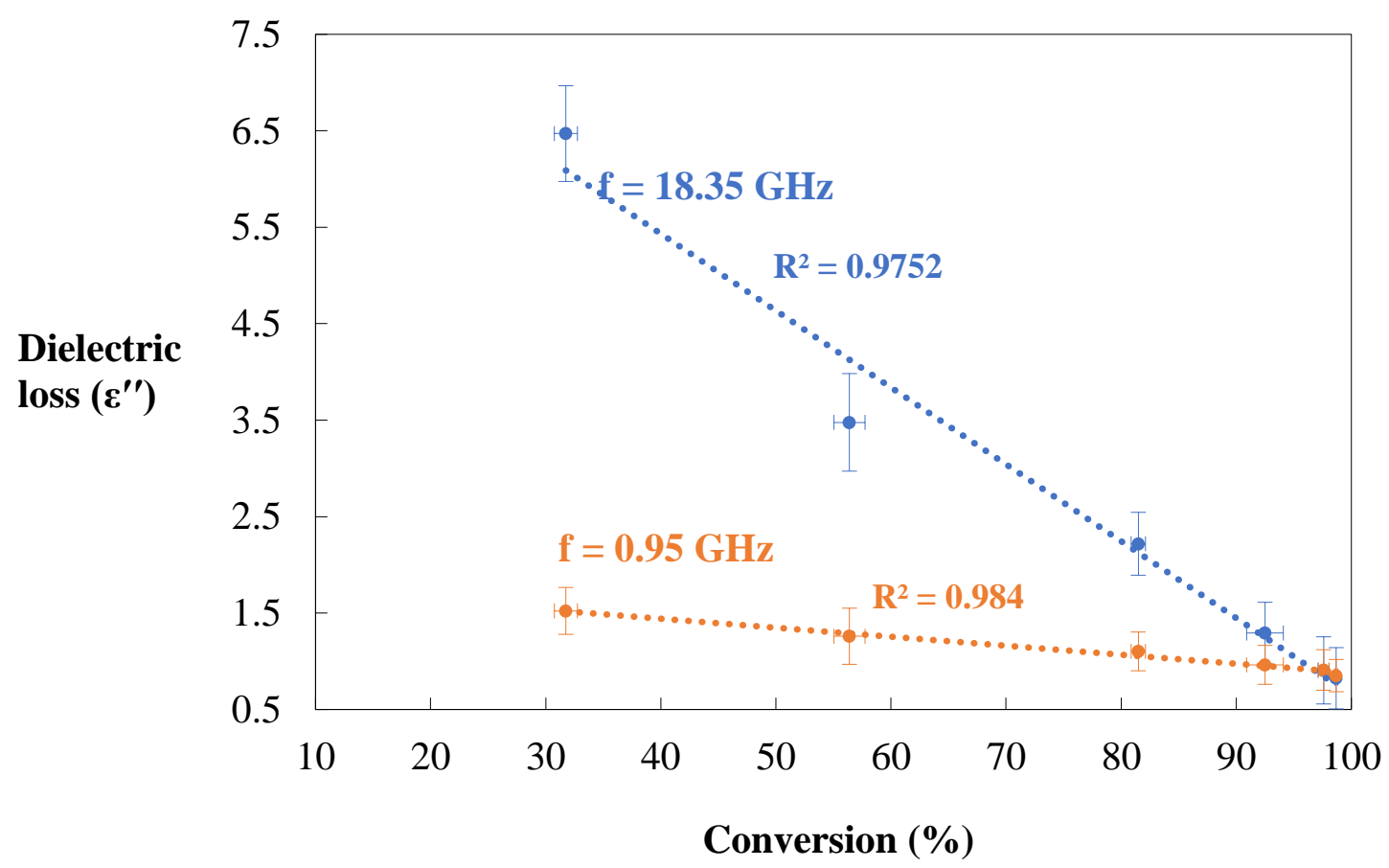

Fig. 9. Graph of dielectric loss $\left(\varepsilon^{\prime \prime}\right)$ versus conversion for frequency $0.95 \mathrm{GHz}$ and 18.35 $\mathrm{GHz}$.

Figures 8 and 9 demonstrate that both $\varepsilon^{\prime}$ and $\varepsilon^{\prime \prime}$ can be linearly related to conversion, as the trend lines exhibit very high coefficient of determination $\left(\mathrm{R}^{2}\right)$ values. Thus, the results obtained in the present study suggest that both $\varepsilon^{\prime}$ and $\varepsilon^{\prime \prime}$ could be used to monitor conversion changes based on the appropriate selection of frequency. However, the use of $\varepsilon^{\prime}$ is still 
observed to be more the appropriate, as it offers a larger differential between the monomer and polymer values and therefore better discrimination during the measurement process. The proposed method can be applied to any polymerisation process that exhibits detectable changes in the dielectric properties over the progress of the reaction. In the particular system which we have chosen to study the dielectric properties of the mixture are dominated by the polar monomer. Therefore, as the polymerisation progresses via the consumption of monomer to create the polymer the dielectric properties of the mixture decrease and this can be detected during the experiment.

\section{Conclusions}

This study has demonstrated the first "on-line" measurement of the dielectric properties of the reaction mixture, during the tin medicated polymerisation of $\varepsilon$-caprolactone. It has clearly demonstrated that this data can be used to identify key reaction parameters, such as the level of monomer to polymer conversion that have been achieved. In detail, both dielectric constant and loss can be used to follow the reaction progress. Furthermore, it was demonstrated that a highly controlled polymerisation could be successfully conducted with the probe immersed within the reaction mixture, as the presence of the probe clearly did not affect the quality of polymerisation achieved. Additionally, the analysis of the dielectric data with frequency allowed the identification of the most suitable monitoring strategy to define the progress of the reaction, by comparing the dielectric data at low and high frequencies. Thus, although it has been shown that the differential in dielectric values is frequency dependent, in the case of $\varepsilon^{\prime}$, both the differentiation between the monomer and polymer responses and the signal to error ratios are sufficiently large that this reaction can be followed across the whole frequently spectrum using an the open ended coaxial probe method. This demonstrated that sensor techniques based on observing the change in dielectric constant using a reflectance method should have broad applicability to many chemical transformations.

Dielectric spectroscopy is a non-destructive monitoring method, does not require sampling and has the potential to be implemented in most polymerisation reactor geometries as a simple retrofit. Therefore, the application of this method has high potential to improve process control strategies and both, optimise product quality and batch to batch repeatability. For example, by clearly identifying the optimum point to terminate the reaction to prevent formation of by-products due to the onset of transesterification at higher conversion or the 
ideal point to add a second monomer to synthesise an architectural polymer. Additionally, it can be used to optimise energy usage by minimising the amount of time that the reaction is kept at the elevated temperatures required for polymerisation. Furthermore, the proposed method was successfully applied in the bulk polymerisation and the significant increase in viscosity that is observed over the course of the reaction has been shown not to influence the quality of the data obtained and it can also aid in the development of processes that focus on eliminating the use of solvents.

\section{Acknowledgements}

The authors would like to thank the Faculty of Engineering at the University of Nottingham for their funding support (A.K.).

\section{References}

[1] C.J. Hawker, A.W. Bosman, E. Harth, Chem. Rev. 101 (2001) 3661-3688.

[2] K. Matyjaszewski, J. Xia, Chem. Rev. 101 (2001) 2921-2990.

[3] A.A. Gridnev, S.D. Ittel, Chem. Rev. 101 (2001) 3611-3660.

[4] H. Deng, Z. Shen, L. Li, H. Yin, J. Chen, J. Appl. Polym. Sci. 131 (2014) n/a-n/a.

[5] Y. Deng, S. Zhang, G. Lu, X. Huang, Polym. Chem. 4 (2013) 1289-1299.

[6] W. Wang, Y. Zheng, E. Roberts, C.J. Duxbury, L. Ding, D.J. Irvine, S.M. Howdle, Macromolecules 40 (2007) 7184-7194.

[7] M.L. Koh, D. Konkolewicz, S. Perrier, Macromolecules 44 (2011) 2715-2724.

[8] Y. Zheng, S. Li, Z. Weng, C. Gao, Chem. Soc. Rev. 44 (2015) 4091-4130.

[9] M. Destarac, Macromolecular Reaction Engineering 4 (2010) 165-179.

[10] F. McLennan, B.R. Kowalski, Process Analytical Chemistry, Springer Science \& Business Media, 1995.

[11] D.C. Hassell, E.M. Bow, Appl. Spectrosc., AS 52 (1998) 18A-29A.

[12] A.M. Alb, M.F. Drenski, W.F. Reed, Polym. Int. 57 (2007) 390-396.

[13] W.-D. Hergeth, in: Ullmann's Encyclopedia of Industrial Chemistry, Wiley-VCH Verlag GmbH \& Co. KGaA, 2000.

[14] K.A. Bakeev, Process Analytical Technology: Spectroscopic Tools and Implementation Strategies for the Chemical and Pharmaceutical Industries, John Wiley \& Sons, 2010. 
[15] I.R. Lewis, H. Edwards, Handbook of Raman Spectroscopy: From the Research Laboratory to the Process Line, CRC Press, 2001.

[16] M.J. Kamaruddin, J.E. Harfi, G. Dimitrakis, N.T. Nguyen, S.W. Kingman, E. Lester, J.P. Robinson, D.J. Irvine, Green Chem. 13 (2011) 1147-1151.

[17] M.J. Kamaruddin, N.T. Nguyen, G.A. Dimitrakis, J.E. Harfi, E.R. Binner, S.W. Kingman, E. Lester, J.P. Robinson, D.J. Irvine, RSC Adv. 4 (2014) 5709-5717.

[18] N.T. Nguyen, E. Greenhalgh, M.J. Kamaruddin, J. El harfi, K. Carmichael, G. Dimitrakis, S.W. Kingman, J.P. Robinson, D.J. Irvine, Tetrahedron 70 (2014) 996-1003.

[19] A.N. Kalamiotis, A.A. Ilchev, D.J. Irvine, G.A. Dimitrakis, in: Proceedings IMCS 2018, AMA Service GmbH, Vienna, Austria, 2018, pp. 763-764.

[20] N. Hill, Dielectric Properties and Molecular Behaviour, Van Nostrand Reinhold Inc.,U.S., London, New York, 1969.

[21] A.C. Metaxas, R.J. Meredith, Industrial Microwave Heating, The Institution of Engineering and Technology, London, UK, 1905.

[22] L.Q. Liao, L.J. Liu, C. Zhang, F. He, R.X. Zhuo, K. Wan, Journal of Polymer Science Part A: Polymer Chemistry 40 (2002) 1749-1755.

[23] M. Labet, W. Thielemans, Chem. Soc. Rev. 38 (2009) 3484-3504.

[24] J.-M. Raquez, R. Narayan, P. Dubois, Macromolecular Materials and Engineering 293 (2008) 447-470.

[25] Keysight Technologies, Agilent 85070E Dielectric Probe Kit $200 \mathrm{MHz}$ to $50 \mathrm{GHz}$ Technical Overview, (2012).

[26] A.M. Nicolson, G.F. Ross, IEEE Transactions on Instrumentation and Measurement 19 (1970) 377-382.

[27] W.B. Weir, Proceedings of the IEEE 62 (1974) 33-36.

[28] A.P. Gregory, R.N. Clarke, IEEE Transactions on Dielectrics and Electrical Insulation 13 (2006) 727-743.

[29] A.P. Gregory, R.N. Clarke, Meas. Sci. Technol. 18 (2007) 1372.

[30] A.P. Gregory, R.N. Clarke, Tables of the Complex Permittivity of Dielectric Reference Liquids at Frequencies Up to $5 \mathrm{GHz}$, National Physical Laboratory, 2012.

[31] P. Dubois, O. Coulembier, J.-M. Raquez, Handbook of Ring-Opening Polymerization, John Wiley \& Sons, 2009.

[32] G. Odian, Principles of Polymerization, John Wiley \& Sons, 2004. 